\title{
The Impact of Adjacent Channel Interference in Multi-Radio Systems using IEEE 802.11
}

\author{
Jens Nachtigall, Anatolij Zubow, Jens-Peter Redlich \\ Humboldt University, Germany. \\ E-mail: \{nachtiga, zubow,.jpr\}@informatik.hu-berlin.de
}

\begin{abstract}
A promising approach for improving the capacity of Wireless Mesh Networks is by making use of multiple non-overlapping RF channels. Multi-channel protocols have the advantage that several devices can transmit in parallel within a collision domain on distinct channels. When using IEEE $802.11 \mathrm{~b} / \mathrm{g} / \mathrm{a}$ most protocol designers assume 3 and 12 non-overlapping channels, respectively. However, this simplified assumption does not hold. We present results from measurements that show that the number of available non-interfering channels depends on the antenna separation, PHY modulation, RF band, traffic pattern and whether single- or multi-radio systems are used. The problem is caused by Adjacent Channel Interference (ACI) where nearby transmitters "bleed over" to other frequencies and either cause spurious carrier sensing or frame corruption. For nearby transceivers, as in the factory defaults of multi-radio devices, this results in at most two noninterfering channels, one within $2.4 \mathrm{GHz}$ and the other within the $5 \mathrm{GHz}$ band. Only if the distance between the antennas is increased, non-interfering channels within the bands themselves become available. Moreover, our comparison of single- and multiradio systems allows us to isolate ACI from board crosstalk and radiation leakage of which only the multi-radio systems seem to suffer. Finally, we show how a packet-level simulator can be improved to realistically incorporate ACI. With the help of this simulator more confident statements about the performance of various multi-channel protocols can be made.
\end{abstract}

Index Terms-Wireless Networks, Multi-Radio, MultiChannel, Cross-Channel, Adjacent Channel Interference, Measurements

\section{INTRODUCTION}

It is known that throughput and latency within a Wireless Mesh Network (WMN) dramatically worsen with the number of hops that need to be traversed for communication [10]. This multi-hop nature is the main difference compared to traditional cellular networks. As WMNs usually operate on the same frequency channel to avoid network partitioning, they also share a common collision domain. That is, while a node transmits a packet to another node within its communication range, all remaining nodes within the much bigger interference range need to remain silent. This has a dramatic impact on the performance of multi-hop networks whose very nature it is to relay other nodes' packets. It is widely assumed that IEEE $802.11 \mathrm{~b} / \mathrm{g}$ offers 3 and 802.11a 12 non-overlapping RF channels. Channels are referred to as being non-overlapping or orthogonal if they can be used in parallel without any negative interference effects. An obvious strategy is to utilize different non-overlapping channels within a WMN in order to split up the collision domain as a way to alleviate the adverse effects of interference. Neighboring nodes can transmit in parallel as long as they use non-overlapping channels. This increases the throughput and reduces the delay significantly. Declining hardware prices boosted this trend: It is not only worthwhile to increase the number of nodes and hence possibly channels of a single-radio, multi-channel network, but also to equip devices with multiple wireless transceivers to establish a socalled multi-radio, multi-channel network.

\section{RELATED WORK}

A significant work was done on protocols that exploit multiple RF channels available in IEEE 802.11 to increase capacity [13]. Most multi-channel protocol designers assume the existence of several non-overlapping channels, e.g. 3 for $802.11 \mathrm{~b} / \mathrm{g}$ and 12 for $802.11 \mathrm{a}$ (FCC domain). While implementing proof-of-concept prototypes of their multi-radio protocols, some authors accidentally stumbled across the fact of Adjacent Channel Interference (ACI) between supposably non-overlapping channels. For instance, Draves et al. [7] could not find any non-interfering channels within $802.11 \mathrm{~b} / \mathrm{g}$ and 802.11a. As a result they had to operate one transceiver in the $2.4 \mathrm{GHz}$ and the other in the $5 \mathrm{GHz}$ band. Of the expected 15 non-interfering frequency channels only two remained. Their theoretical multi-radio routing metric was effectively reduced to a two-radio routing metric. Adya et al. [1] propose a link layer protocol operating on distinct frequency channels. Only by separating the radios by at least $30 \mathrm{~cm}$ three non-interfering channels became available for $802.11 \mathrm{~b} / \mathrm{g}$. They also noted that ACI is highly hardware dependent. Robinson et al. [16] used a more systematical measurement methodology. However, they used rather untypical hardware for WMNs - Dell workstations with four PCI slots. They observed that merely plugging an additional wireless card into a workstation and operating it in passive monitor mode can reduce the throughput. They accounted this to board crosstalk and radiation leakage of the passive cards. In contrary to our results, they found that even two receiving radios were strongly interfering with each other. It should be noted that they sent 802.11 unicast packets that require acknowledgments in the opposite direction, whereas we sent broadcast packets only. Therefore, Robinson et al. did not isolate the different traffic patterns of receiving and transmitting as clearly from each other as we did. However, they also observed the need to increase the distance between the radios, in their case $1 \mathrm{~m}$, to get at least two non-overlapping channels within $802.11 \mathrm{~b} / \mathrm{g}$. The same was emphasized in [4], 
[14] and [12], whereas the latter required a distance of at least $1 \mathrm{~m}$ between their omni-directional $5 \mathrm{dBi}$ antennas for a fixed bitrate of 11 Mbps. Cheng et al. [5], [6] presented results for 802.11a. However, they only considered three neighboring channels within 802.11a. The most recent work on ACI can be found in [8]. Four Intel laptops (with 2200BG Mini-PCI cards) formed two links, whose two ends were close to each other. One link was set to channel 3 of $802.11 \mathrm{~b}$ and the other to channel 8 . Surprisingly, the radio on channel 3 could even decode packets from channel 8.

\section{THEORY}

In this chapter we explain why two distinct channels might interfere with each other. The notion of Adjacent Channel Interference is most important. An additional problem, socalled Board Crosstalk or Radiation Leakage occurs when using multi-radio systems. Finally, the number of non-interfering channels also depends on the used radio spectrum.

\section{A. Interference}

\section{Definition 1: Adjacent Channel Interference}

Interference that is caused by nearby transmitters on distinct frequency channels "bleeding over" to another channel is called adjacent channel interference (ACI) [15, p. 74].

The resulting phenomenons are commonly referred to as the near-far or near-field effect [11, p. 279]. For 802.11, one can distinguish between the following consequences (to be discussed more deeply in Sec. IV): For the case of two nearby transmitters (referred to as TX-TX) the overlapping ACI of one transmitter causes a spurious carrier sensing at the other. Remember that 802.11 is a CSMA/CA protocol which follows the listen-before-talk paradigm. That means that a station is only allowed to transmit if the medium is idle. ACI may trigger the carrier sensing mechanisms to report that the medium is busy. In this case the station will misleadingly defer its transmission. For the case of a receiver and a transmitter on distinct channels in close proximity (RX-TX) the weak incoming signal at the receiver gets corrupted by the strong outgoing signal of the nearby transmitter. Both consequences are also described in the measurement based studies of [8] and [5]. We also observed a problem for two receiving nodes in close vicinity $(\mathrm{RX}-\mathrm{RX})$ : The ACI causes a variant of the hidden-node problem that cannot be tackled with RTS/CTS since the two links are on distinct channels and therefore the receiver is unable to decode the NAV value from the RTS/CTS packets.

Throughout our evaluation we considered two different device types: single- and multi-radio systems. The latter refers to devices equipped with multiple radio transceivers. In contrast, single-radio systems have only one radio transceiver. When using multi-radio systems an additional problem appears, the so-called board crosstalk or radiation leakage:

\section{Definition 2: Board Crosstalk and Radio Leakage}

Board crosstalk is defined as noise caused by the usage of a common bus by several WiFi cards. On the other hand, radiation leakage refers to over-the-air interference due to imperfect shielding of the WiFi cards [16].
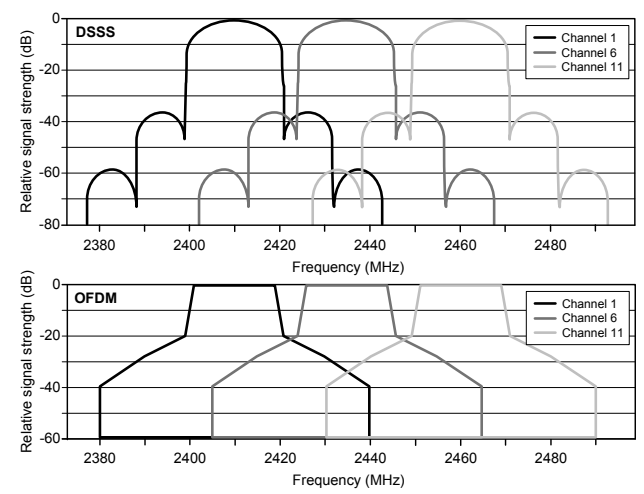

Figure 1. Channel separation in 802.11 b DSSS (top) and 802.11g OFDM (bottom). Adapted from [9].

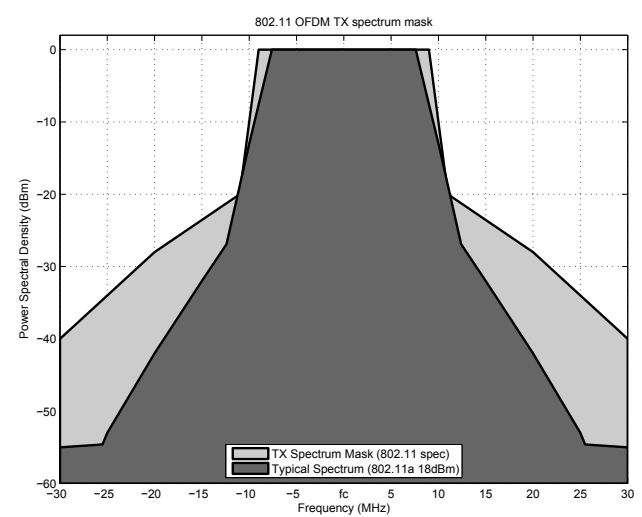

Figure 2. OFDM transmit mask as required by $802.11 \mathrm{~g} / \mathrm{a}$ (outer line) compared with a real transmission at $18 \mathrm{dBm}$ measured with a spectrum analyser (inner line) [6].

Since we used both single-radio as well as multi-radio systems, we could isolate the interference effects caused by board crosstalk or radiation leakage from ACI.

\section{B. Radio Spectrum Usage}

a) $802.11 \mathrm{~b} / \mathrm{g}(2.4 \mathrm{GHz})$ : The DSSS PHY has at most 13 channels in the $2.4 \mathrm{GHz}$ band, each $5 \mathrm{MHz}$ wide. Channel 1 is placed at $2.412 \mathrm{GHz}$, channel 2 at $2.417 \mathrm{GHz}$, and so on up to channel 13 at $2.472 \mathrm{GHz}$. DSSS is a single-carrier modulation. Within a channel, most of the signal energy is spread across a 22-MHz band. To prevent interference to adjacent channels, the first side lobe is filtered to $30 \mathrm{~dB}$ below the power at the channel center frequency. Additional lobes are filtered to $50 \mathrm{~dB}$ below the power at the channel center [9]. ACI influences the number of channels that can be used simultaneously. The IEEE 802.11 b specifies that $25 \mathrm{MHz}$ spacing is sufficient. Fig. 1 (top) shows the spectral mask of transmissions on the so-called non-overlapping channels $(1,6$, and 11). The multi-carrier OFDM PHY is also available in the $2.4 \mathrm{GHz}$ band. As with the DSSS PHYs, a transmit mask limits power leakage into the side bands. Fig. 2 shows the transmission mask as required by $802.11 \mathrm{~g} / \mathrm{a}$ and a real OFDM transmission measured with a spectrum analyser. Fig. 1 (bottom) shows the spectral mask of OFDM on the so-called non-overlapping channels. 


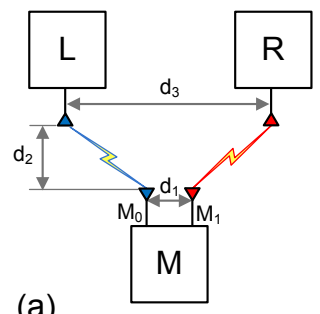

(a)

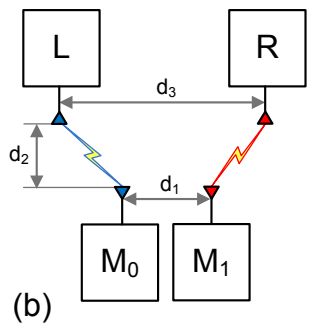

Figure 3. Node placement: (a) multi-radio $M$ with interfaces $M_{0}$ and $M_{1}$ (b) two single radios $M_{0}$ and $M_{1}$

b) $802.11 \mathrm{a}(5 \mathrm{GHz})$ : The OFDM PHY in the $5 \mathrm{GHz}$ band offers eight channels for indoor and eleven for outdoor use (ETSI domain), each $20 \mathrm{MHz}$ wide. In comparison to the $2.4 \mathrm{GHz}$ band, the channel spacings are larger -20 instead of $5 \mathrm{MHz}$ [9].

\section{MeAsurements}

The measurements were taken on the topology depicted in Fig. 3. Three nodes referred to as left $(L)$, middle $(M)$ or right $(R)$ node were put indoors in an almost straight line. The left and right node were single-radio devices, whereas we considered two different setups for the middle node: At first a multi-radio system equipped with two radio transceivers (Fig. 3a) was used to evaluate the impact of both ACI as well as board crosstalk and radiation leakage. To isolate both effects from each other we also replaced the multi-radio device in the middle by two single-radio devices (Fig. 3b). Five different distances were used for $d_{1}(15,40,80,160$ and $320 \mathrm{~cm})$, of which a separation of $15 \mathrm{~cm}$ corresponds to the factory defaults. Distances $d_{2}$ and $d_{3}$ were kept fixed at 140 and $1020 \mathrm{~cm}$. We used WRAP.2E boards ${ }^{1}$ (233 MHz AMD Geode x86 CPU, 128MB RAM) equipped with two Mini-PCI slots together with Routerboard R52 wireless IEEE $802.11 \mathrm{a} / \mathrm{b} / \mathrm{g}$ combo cards (Atheros AR5414 chipset) ${ }^{2}$. All boards installed in waterproof outdoor metal cases $^{3}$ were placed $26 \mathrm{~cm}$ above the ground and had clear line of sight to each other. Omnidirectional dual-band antennas with a gain of $5 \mathrm{dBi}$ were mounted with equal vertical polarization. Unless the default antenna separation of the WRAP-Board $(15 \mathrm{~cm})$ was used, we added an RF cable (HDF-200) of $150 \mathrm{~cm}$ length to each connector for the multi-radio case in order to increase the antenna separation at the middle node to up to $320 \mathrm{~cm}$. The RF cable induced an additional attenuation of about $2 \mathrm{~dB}$. On the software side we chose OpenWrt ${ }^{4}$ version 7.09 with Linux kernel 2.6.22 as operating system and MadWifi ${ }^{5}$ version 0.9.3 as WiFi driver. MadWifi's regdomain setting was changed to the ETSI and countrycode to Germany in order to obtain 13 channels for $802.11 \mathrm{~b} / \mathrm{g}$. Antenna Diversity was disabled and only the main connector used. The packet generation and capturing was done with the Click Modular Router ${ }^{6}$

${ }^{1}$ WRAP.2E boards: http://www.pcengines.ch/wrap2e3.htm

${ }^{2}$ R52 cards: http://www.mikrotik.com/pdf/R52.pdf

${ }^{3}$ Metal cases: http://www.mini-box.com/WRAP-BOX-2A1E

${ }^{4}$ OpenWrt Linux distribution for embedded devices: http://openwrt.org

${ }^{5}$ Multimode Atheros Driver for WiFi on Linux: http://madwifi.org

${ }^{6}$ The Click Modular Router: http://www.read.cs.ucla.edu/click/

\begin{tabular}{ll}
\hline Parameter & Value \\
\hline Systems & Single- and two-radio devices \\
Scenarios & TX-TX $\left(M_{0}, M_{1}\right.$ transmitting $)$, \\
& RX-RX $\left(M_{0}, M_{1}\right.$ receiving $)$, \\
& RX-TX $\left(M_{0}\right.$ receiving, $M_{1}$ transmitting) \\
Antenna separations & $15,40,80,160,320 \mathrm{~cm}$ \\
Physical layer & $802.11 \mathrm{a} / \mathrm{b} / \mathrm{g}$ \\
Transmission power & $6(\mathrm{RX}-\mathrm{TX}$ only $), 16 \mathrm{dBm}$ \\
Bitrates & $1 \mathrm{Mbps}(\mathrm{DSSS}), 6 \mathrm{Mbps}(\mathrm{OFDM})$ \\
Radio frequencies & $2.4 \mathrm{GHz}(\mathrm{ch} .1-13), 5 \mathrm{GHz}(\mathrm{ch} .36-64)$ \\
RTS/CTS & Disabled \\
WiFi frame size & $1500 \mathrm{bytes}$ \\
Transmission mode & Broadcast \\
Flow duration & 10 sec \\
Number of runs & 10 \\
& \multicolumn{1}{c}{ Table I } \\
& MEASUREMENTS PARAMETERS
\end{tabular}

software version 1.5. During transmission and reception we monitored that the CPU load remained within safe grounds and did not become the bottleneck. The interference due to external networks was negligible. Our scripts and all dump files (PCAP) are available on our website for further analysis ${ }^{7}$.

The parameters we used throughout our measurements are summarized in Table I. We considered the following three scenarios: i) The middle node transmits on both of its interfaces (TX-TX). The left node is set to receiving packets from $M_{0}$, the right from $M_{1}$. For non-interfering channels one would expect that both radios in the middle can transmit in parallel. So the total throughput should be doubled compared to setups were both radios are on the same channel. ii) Both interfaces in the middle receive packets from left and right (RX-RX). Since two flows can be received simultaneously, one would again expect the throughput to double. iii) The left node sends packets to $M_{0}$ while at the same time $M_{1}$ transmits to the right node (RX-TX). This is the full-duplex case and for non-interfering channels one would expect the throughput to remain stable and not halved as seen for single-channel systems.

We considered both the DSSS and the OFDM physical layers with a bitrate of 1 and $6 \mathrm{Mbps}$, respectively. Broadcast WiFi frames with a size of $1500 \mathrm{~B}$ were sent out as fast as possible. Since acknowledgments were not used, we were able to obtain strictly directive flows. For all experiments the link between $L$ and $M_{0}$ was fixed at channel 1 for $802.11 \mathrm{~b} / \mathrm{g}$ and 36 for $802.11 \mathrm{a}$, respectively, while the channel for the link between $M_{1}$ and $R$ was varied (Fig. 3). For each scenario and channel assignment the experiment lasted 10 seconds and was repeated 10 times. Beforehand, the links were independently measured to ensure that the signal is strong enough and the Packet Error Rate (PER) zero for all links (baseline tests).

\section{A. TX-TX Scenario}

In this section we evaluate channel orthogonality for two transmitting radios in close vicinity. We setup two flows: from $M_{0}$ to $L$ and $M_{1}$ to $R$ (Fig. 3).

Fig. 4 shows the results for a multi-radio system with DSSS PHY of $1 \mathrm{Mbps}$ for the distance of $15 \mathrm{~cm}$. This is the default

${ }^{7}$ http://sartrac.informatik.hu-berlin.de/channel-orthogonality 


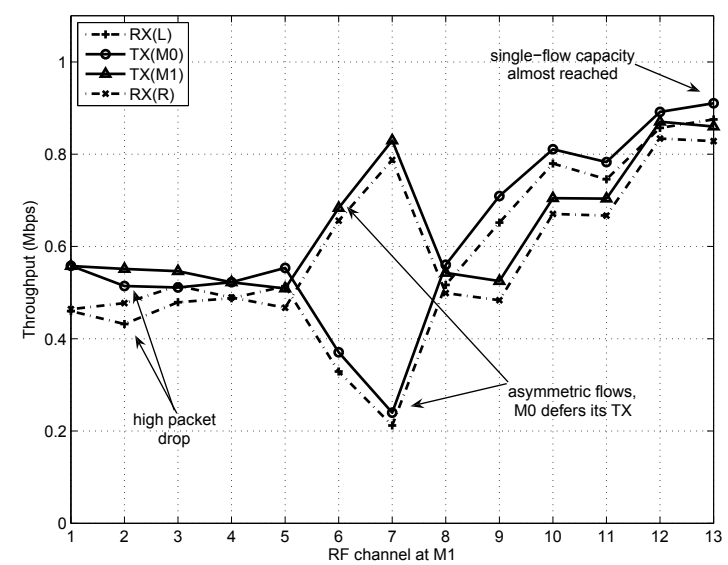

Figure 4. TX-TX scenario (multi-radio, DSSS PHY, $15 \mathrm{~cm}, 2.4 \mathrm{GHz}$ ).

antenna separation without additional RF cables. From channel $1-5$ both transmitters equally share the medium as would be expected from a fair CSMA/CA protocol. From channel 6-8 the total throughput remains the same, however, one of the transmitting nodes $\left(M_{0}\right)$ defers its transmission in favor of the other node $\left(M_{1}\right)$. This may be due to spurious carrier sensing at node $M_{0}$ in this range of low ACI received from nearby $M_{1}$, whereas $M_{1}$ seems to sense the ACI from $M_{0}$ less strong. From channel 9-13 the total throughput constantly increases as the ACI decreases. Hence, the medium is not sensed busy anymore and a maximum of about 0.9 Mbps is reached at channel 12. For farther antenna separations we achieved a maximum of about $1 \mathrm{Mbps}$. This suggests that the throughput for the setup in Fig. 4 would still increase if additional channels were available. Notice that there is always a packet drop of about $10 \%$ on both links which is neither present if only one link is active as for the baseline tests nor for the single-radio setups. Board crosstalk or radiation leakage may be the reason for that (see Def. 2).

The above situation $(15 \mathrm{~cm})$ improves if $d_{1}$ is increased to $160 \mathrm{~cm}$ or more. The total throughput reaches its maximum of $1 \mathrm{Mbps}$ at channel 6 for a antenna separation of $160 \mathrm{~cm}$ and for $320 \mathrm{~cm}$ already at channel 5 . The results are summarized in Table II (col. "DSSS 2.4 GHZ" at TX-TX). The "asymmetric flow gap" of Fig. 4 is shifted to the left for those higher distances, that is, towards nearer channels since a lower ACI is reached sooner.

Fig. 5 shows the results for the same setup, but using the OFDM PHY of 6 Mbps. Here, the total throughput remains almost the same for all channels. This is in contrast with DSSS PHY where at channel 12 for the second radio $\left(M_{1}\right)$ the total throughput almost doubled (Fig. 4). By increasing the distance between the antennas the situation also improves for OFDM. With a separation of $40 \mathrm{~cm}$ channels 1 and 8 become non-interfering (Table II, col. "OFDM 2.4 GHZ” at TX-TX). For the multi-radio system the packet drops are smaller with OFDM PHY than with DSSS PHY, but again still present in contrast to the single-radio setups.

The results of the multi-radio setup in the $5 \mathrm{GHz}$ band (OFDM) are depicted in Fig. 6. When the radios are spatially nearby as with the factory defaults of $15 \mathrm{~cm}$, then only two

\begin{tabular}{|c|c|c|c|c|}
\hline Scenario & $\begin{array}{l}\text { Antenna } \\
\text { separation }\end{array}$ & $\begin{array}{l}\text { DSSS } \\
2.4 \mathrm{GHz}\end{array}$ & $\begin{array}{l}\text { OFDM } \\
2.4 \mathrm{GHz}\end{array}$ & $\begin{array}{l}\text { OFDM } \\
5 \mathrm{GHz}\end{array}$ \\
\hline \multirow{5}{*}{$\begin{array}{l}\underset{x}{x} \\
\stackrel{x}{E}\end{array}$} & $15 \mathrm{~cm}$ & $12(12)$ & $\mathrm{n} / \mathrm{a}(\mathrm{n} / \mathrm{a})$ & $60(48)$ \\
\hline & $40 \mathrm{~cm}$ & 9 & 8 & 48 \\
\hline & $80 \mathrm{~cm}$ & 7 (7) & $6(8)$ & 44 (44) \\
\hline & $160 \mathrm{~cm}$ & 6 & 6 & 44 \\
\hline & $320 \mathrm{~cm}$ & $5(5)$ & $6(6)$ & $44(44)$ \\
\hline \multirow{5}{*}{$\frac{\underset{x}{x}}{\underline{x}}$} & $15 \mathrm{~cm}$ & $5(5)$ & $5(5)$ & $44(44)$ \\
\hline & $40 \mathrm{~cm}$ & 5 & 6 & 44 \\
\hline & $80 \mathrm{~cm}$ & $5(5)$ & $5(6)$ & 44 (44) \\
\hline & $160 \mathrm{~cm}$ & 5 & 6 & 44 \\
\hline & $320 \mathrm{~cm}$ & $5(5)$ & $5(6)$ & $44(44)$ \\
\hline \multirow{5}{*}{ 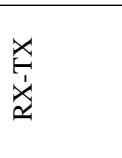 } & $15 \mathrm{~cm}$ & n/a (n/a) & n/a (n/a) & $\mathrm{n} / \mathrm{a}(48)$ \\
\hline & $40 \mathrm{~cm}$ & 5 & $\mathrm{n} / \mathrm{a}$ & 52 \\
\hline & $80 \mathrm{~cm}$ & $5(6)$ & n/a (n/a) & $44(48)$ \\
\hline & $160 \mathrm{~cm}$ & 5 & 9 & 48 \\
\hline & $320 \mathrm{~cm}$ & $6(5)$ & $9(11)$ & 48 (44) \\
\hline
\end{tabular}

MeAsurement Results of ALl SCENARIOS: The $1^{\text {ST }}$ NUMBER IS THE NEAREST NON-INTERFERING CHANNEL ON $M_{1} \rightarrow R$ FOR THE MULTI-RADIO, THE NUMBER IN BRACKETS FOR THE CORRESPONDING SINGLE-RADIO SETUP. THE LEFT LINK $\left(L \rightarrow M_{0}\right)$ WAS FIXED AT CHANNEL 1 FOR $802.11 \mathrm{~B} / \mathrm{G}$ AND 36 FOR $802.11 \mathrm{~A}$

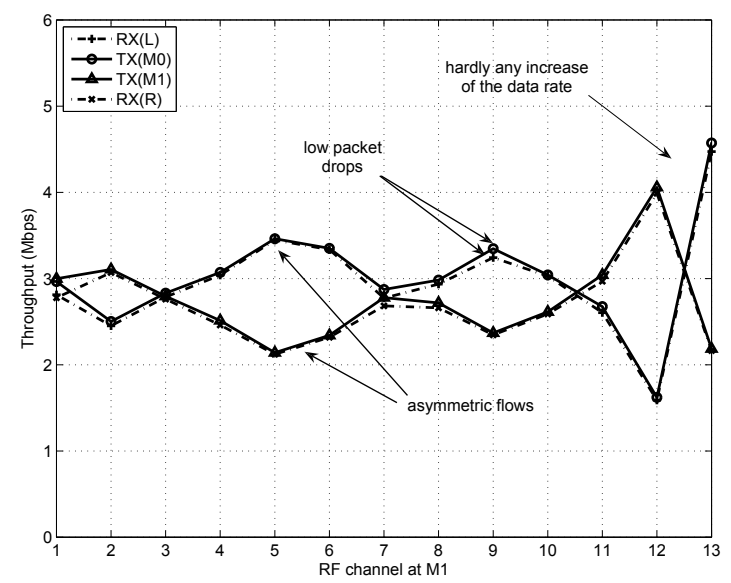

Figure 5. TX-TX scenario (multi-radio, OFDM PHY, $15 \mathrm{~cm}, 2.4 \mathrm{GHz}$ ).

non-interfering channels are available: 36 and at nearest 60 . This is surprising as the $5 \mathrm{GHz}$ band for indoor use offers a bandwidth of $140 \mathrm{MHz}$. As the distance between the two antennas is increased to $80 \mathrm{~cm}$ the channels 36 and 44 become non-interfering for the multi-radio system. The results are better for the single-radio system. Although the two radios are still only separated by $15 \mathrm{~cm}$ channels 36 and 48 can now be used simultaneously (Table II, col. "OFDM 5 GHZ" at TX-TX).

\section{B. $R X-R X$ Scenario}

Next we evaluate channel orthogonality for two receiving radios in close vicinity.

In Fig. 7 the results are displayed for DSSS PHY where the antennas' separation was set to $15 \mathrm{~cm}$. The maximum throughput of $1 \mathrm{Mbps}$ per flow is reached at channel 5. Further, when using channels $2-4$ the packet flow is asymmetric. At channel 1 and 2 the PER is about $11 \%$. We noticed a strange result at channel 12. At this channel the observed throughput was very low for some setups. It looks like there is a MadWifi 


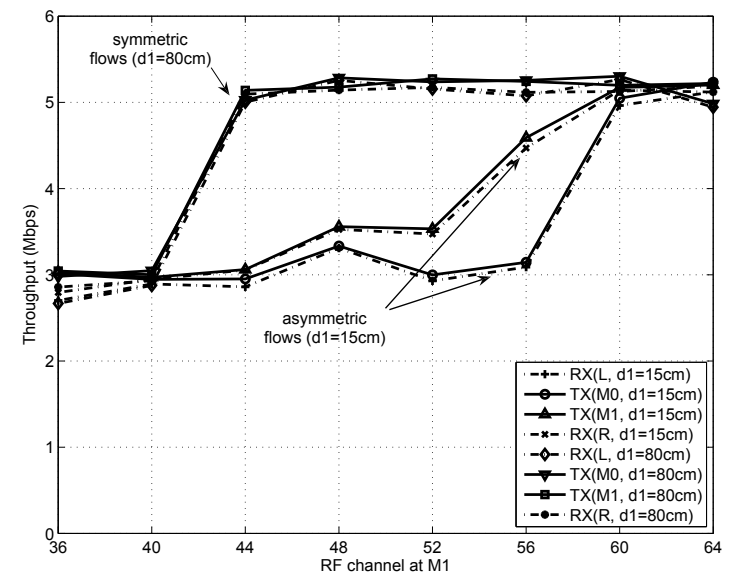

Figure 6. TX-TX scenario (multi-radio, OFDM PHY, 15 and $80 \mathrm{~cm}, 5 \mathrm{GHz}$ ).

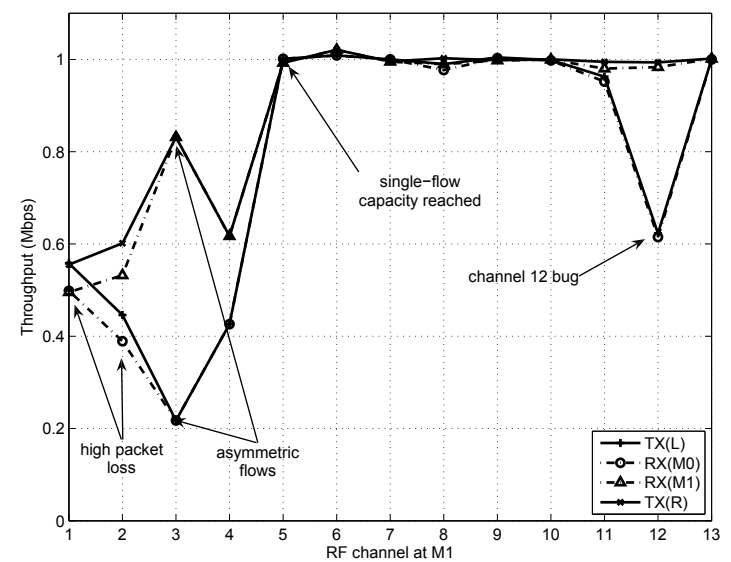

Figure 7. RX-RX scenario (multi-radio, DSSS PHY, $15 \mathrm{~cm}, 2.4 \mathrm{GHz}$ ).

driver bug at this channel. We replaced the hardware, but the problem remained. This only occurs with DSSS PHY. Increasing the distance between the two receiving radios does not yield better results - channel 5 remains the closest noninterfering channel (Table II, col. "DSSS 2.4 GHz" at RX-RX).

Fig. 8 shows the results for OFDM PHY in the $2.4 \mathrm{GHz}$ band. As with DSSS PHY we have a high packet loss at channels 1 and 2. We observed simular PERs for other setups with very close channel assignments where the carrier sensing should actually trigger more reliably. This suggests that a rather aggressive CCA policy is used by Atheros, since the other link is on the same or very nearby channel. At channel 3 4 there is no packet loss. For the channels 4-6 the performance suffers again due to packet drops. We believe that this is now a variant of the hidden node problem - nodes $L$ and $R$ do not sense each other's ACI anymore and go on transmitting which leads to collisions at the middle node $M$. This type of hidden node problem results from ACI and is therefore different from the well-known cases due to co-channel interference. Even RTS/CTS cannot counteract this as an interfering radio on an adjacent channel would not decode it. For higher channel separations (7-13) the high packet loss of about $10 \%$ for each flow remains whereas it only amounts to $2 \%$ for the same setup with single-radio devices. As with DSSS PHY a

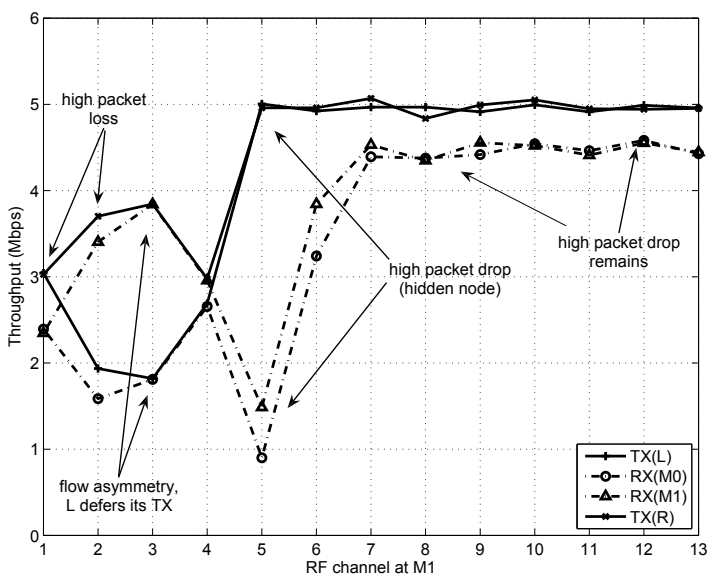

Figure 8. RX-RX scenario (multi-radio, OFDM PHY, $15 \mathrm{~cm}, 2.4 \mathrm{GHz}$ ).

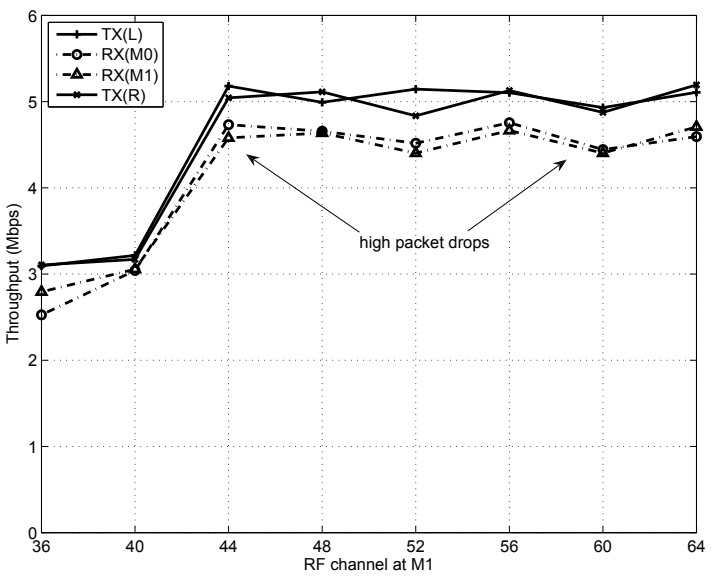

Figure 9. RX-RX scenario (multi-radio, OFDM PHY, $15 \mathrm{~cm}, 5 \mathrm{GHz}$ ).

further increase in the antenna separations of the middle node (e.g. $320 \mathrm{~cm}$ ) does not make nearer frequencies than channel 5 available for simultaneous use (Table II, col. "OFDM 2.4 GHz” at RX-RX).

At last, we also show the effects of using closely separated radios in the $5 \mathrm{GHz}$ band (Fig. 9). Channel 36 and 44 are nonoverlapping. However, there is always a high PER of about $9 \%$ for each flow. This seems to be a board crosstalk or radiation leakage problem as the drops do not occur when using two single-radios.

\section{RX-TX Scenario}

Here we look at channel orthogonality when one radio is receiving while the other one is transmitting. This is a very important setup as some multi-channel protocols use multiradio systems to operate in full-duplex mode. We setup the packet flow from $L$ to $M_{0}$ and $M_{1}$ to $R$. This section is divided into two parts. At first we consider the case where the received signal is strong in term of signal strength. Here, all radios transmit with $16 \mathrm{dBm}$. In the second part we decreased the transmit power of node $L$ to $6 \mathrm{dBm}$. The idea here is to simulate links of real-world mesh networks which are often weak [3]. 


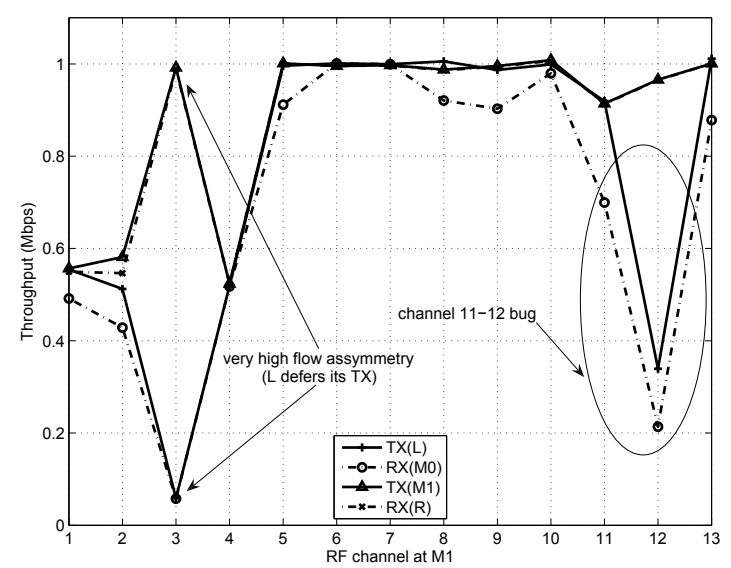

Figure 10. RX-TX scenario (multi-radio, DSSS PHY, $15 \mathrm{~cm}, 2.4 \mathrm{GHz}$ ). Radio $L$ is transmitting with $16 \mathrm{dBm}$.

Let us start with the case where all devices transmit with $16 \mathrm{dBm}$. Again, we first consider the default antenna separation of $15 \mathrm{~cm}$ between the two middle radios $M_{0}$ and $M_{1}$. Fig. 10 illustrates the results. Channels 1 and 5 are noninterfering. For channels 1 and 2 we again notice a PER of about $12 \%$ at node $M_{0}$. At channel 3 the total throughput remains the same, however, the flows are very asymmetric as we have already seen for other scenarios like TX-TX in Fig. 4. There are significant packet drops, e.g. at channels 5, 8 and 9. The bug at channel $11-12$ is again present. The results do not change when the distance between the two middle radios is increased.

The performance of OFDM in the $2.4 \mathrm{GHz}$ band is depicted in Fig. 11. The most interesting fact here is that we cannot find any two non-interfering channels even with the strong transmission power at the left node. Even when using channel 13 a PER of $41 \%$ remains for the link between $L$ and $M_{0}$. The situation improves if we increase the distance between the antennas to $320 \mathrm{~cm}$. From channel 7 onwards the negative consequences of the near-far effect are mitigated, although the loss on the left link $\left(L \rightarrow M_{0}\right)$ remains high with about $8 \%$ compared to the lossless right link $\left(M_{1} \rightarrow R\right)$. The effects of ACI diminish when switching to the $5 \mathrm{GHz}$ band. Here channels 36 and 52 are non-interfering for the distance of only $15 \mathrm{~cm}$. By increasing the distance between the two radios to $320 \mathrm{~cm}$ channel 48 can be used.

Next, the transmitting power of node $\mathrm{L}$ was reduced to 6 $\mathrm{dBm}$ to simulate more real-life links. Note that the PER was still zero for this weak link in the baseline tests. We start with the case where the two radios are spatially close to each other $(15 \mathrm{~cm})$. Fig. 12 illustrates the results. Between channel 2 and 4 CSMA/CA again does not seem to be able to guarantee for a fair sharing of the medium. The left node defers its transmission in favor of $M_{1}$. From channel 5 onwards $L$ does not seem to backoff anymore, presumably because the ACI from $M_{1}$ does not trigger its CCA anymore. The results are even worse than in the TX-TX scenario where the transmission of packets was only deferred due to spurious CCA at the MAC layer. But here the left node sends packets and therefore seizes the medium so that all other nodes within its neighborhood

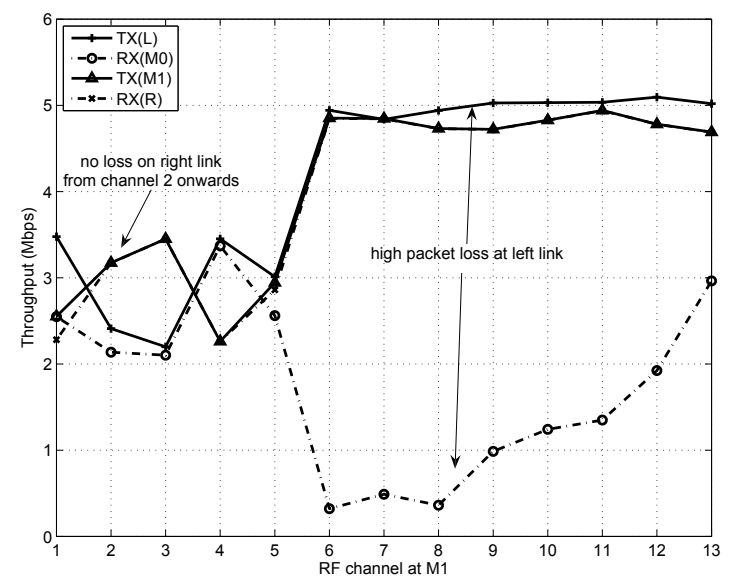

Figure 11. RX-TX scenario (multi-radio, OFDM PHY, $15 \mathrm{~cm}, 2.4 \mathrm{GHz}$ ). Radio $L$ is transmitting with $16 \mathrm{dBm}$.

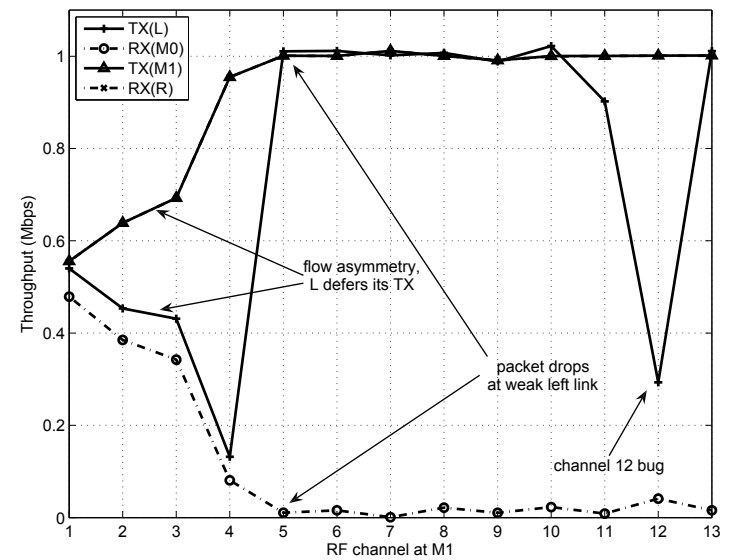

Figure 12. RX-TX scenario (multi-radio, DSSS PHY, 15 cm, $2.4 \mathrm{GHz}$ ). Radio $L$ is transmitting with $6 \mathrm{dBm}$.

cannot transmit at the same time. Moreover, the packets sent by the left node are almost always corrupted at $M_{0}$ due to the strong output power of the transmitting radio $M_{1}$. This requires retransmission at node $L$ along with exponential backoffs. Hence, for RX-TX the problem is at the PHY layer.

Increasing the distance between $M_{0}$ and $M_{1}$ helps to counteract the near-far problem (Table II, col. "DSSS $2.4 \mathrm{GHz}$ " at RX-TX). The transmitting radio $M_{1}$ is now far enough separated from the receiving antenna $M_{0}$. Due to the pathloss attenuation the disrupting ACI of the transmitting radio is now weak enough at the receiving radio, so that it cannot be harmfull anymore. However, even for such a high separation the sharing of the medium remains asymmetric at channel 2-4.

When using OFDM PHY in $2.4 \mathrm{GHz}$ we get slightly worse results as with DSSS PHY (Table II, col. "OFDM $2.4 \mathrm{GHz}$ " at RX-TX). An interesting observation can be made in the 5 $\mathrm{GHz}$ band (Fig. 13). Only the received rate at $M_{0}$ is illustrated now, while the transmission rates at $L$ and $M_{1}$ were always at maximum from channel 44 onwards and shared at channel 36 and 40 . For the nearest distance of $15 \mathrm{~cm}$ interference is present at all available channels. For a separation of $160 \mathrm{~cm}$ the effects of ACI are mitigated from channel 52 onwards. The 


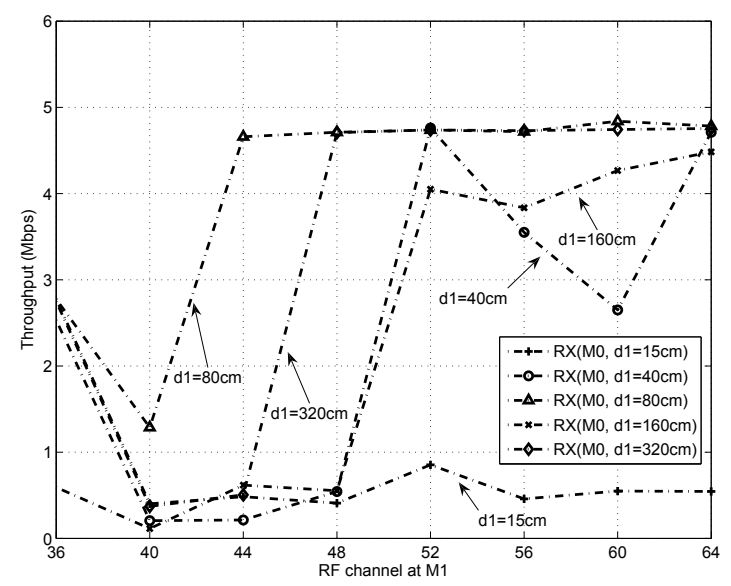

Figure 13. RX-TX scenario (multi-radio, OFDM PHY, 15 and $320 \mathrm{~cm}$, $5 \mathrm{GHz}$ ). Radio $L$ is transmitting with $6 \mathrm{dBm}$.

same holds true for the distance of $40 \mathrm{~cm}$. For $320 \mathrm{~cm}$ the nearfar effect diminishes already from channel 48 onwards and for $80 \mathrm{~cm}$ even from channel 44 onwards. The order of distances is not fully logical as additional effects like multi-path might play a role.

\section{Simulation}

In this section we describe the modifications we made to our packet-level simulator in order to support multiple channels. Our aim was to find a very realistic model which also incorporates ACI. Throughout this section we consider single-radio systems using $802.11 \mathrm{~g}$.

\section{A. Model}

JiST/SWANS [2] was used as packet-level simulator. We extended the radio model to support multiple overlapping channels. In the initial version of JiST/SWANS the radio of each node is modeled as a finite state machine (Fig. 14a). In a first step we changed this by increasing the number of radios belonging to a node from 1 to 13 - one for each channel in case of the $2.4 \mathrm{GHz}$ band. These 13 radios per node now run in parallel. An incoming RX signal is classified according to its annotated channel and directed to the radio with the same channel (Fig. 14b). In a second step we implemented the ACI. As an example, consider a packet which was sent on channel 3. Our observation from the measurements was that not only nodes operating on channel 3 may receive or be interfered by that signal, but also nodes operating on neighboring channels (e.g. 2 and 4) are affected. To correctly model this behavior we have to take a closer look at a typical transmit spectrum mask of an $802.11 \mathrm{~g}$ transmission. Fig. 2 shows the theoretical transmission mask according to $802.11 \mathrm{~g} / \mathrm{a}$ as well as a transmission mask from a real $802.11 \mathrm{~g} / \mathrm{a}$ OFDM transmission at $18 \mathrm{dBm}$ measured with a spectrum analyzer [6]. Remember that the channel spacing is $5 \mathrm{MHz}$. A receiver operating on channel 5 instead of 3 will receive that signal with reduced signal strength of $-13 \mathrm{~dB}$. We used this observation to model ACI. Therefore, for each incoming packet we calculate the signal strength on all neighboring channels according to Table III.

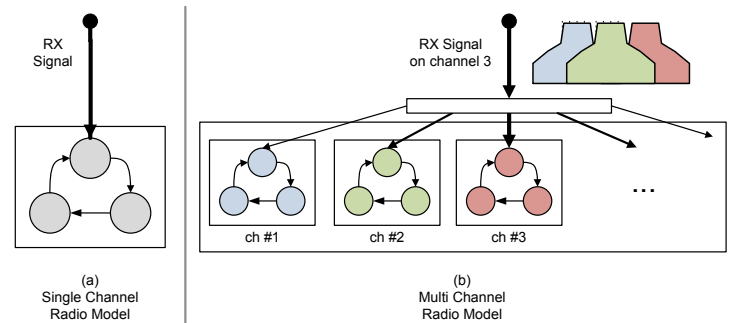

Figure 14. The modified radio model for multiple overlapping RF channels.

\begin{tabular}{lrrrrrr} 
Frequency offset $(\mathbf{M H z})$ & 5 & 10 & 15 & 20 & 25 & 30 \\
\hline Spectral attenuation (dB) & 0 & -13 & -32 & -42 & -53 & -55 \\
& Table III & & & &
\end{tabular}

NUMMERICAL VALUES OF THE TYPICAL TRANSMIT MASK OF FIG. 2.

Then these signals are simultaneously processed by the radios running in parallel. As an example, consider Fig. 14b. Here a signal is sent on channel 3. This signal is passed on unmodified to the radio on channel 3 . The signal is also passed unmodified to the radios on the neighboring channels 2 and 4 . However, for channels 1 and 5 the signal is reduced by $-13 \mathrm{~dB}$. The remaining channels are modified according to Table III.

\section{B. Results}

Fig. 15 illustrates the simulation results for the TX-TX scenario. Here, $F_{1}$ and $F_{2}$ represent the flows on the links $L \rightarrow M_{0}$ and $M_{1} \rightarrow R$. That is, we only consider the total throughput of a link and do not differentiate between sent and received packets anymore. For the short distance of $15 \mathrm{~cm}$ only the channels 1 and 8 are non-interfering. By increasing the distance to $160 \mathrm{~cm}$ we can use channel 6 . Channels 1 and 5 are non-interfering when the distance becomes greater than $500 \mathrm{~cm}$. For most setups of the RX-RX scenario channels 1 and 4 are non-interfering (Fig. 16). However, if we increase the distance between both senders significantly $(20 \mathrm{~m})$, channel 1 and 3 can be used at the same time. Finally, consider the RX-TX scenario depicted in Fig. 17. The flows are very asymmetric for distances of 15 and $40 \mathrm{~cm}$. In the former

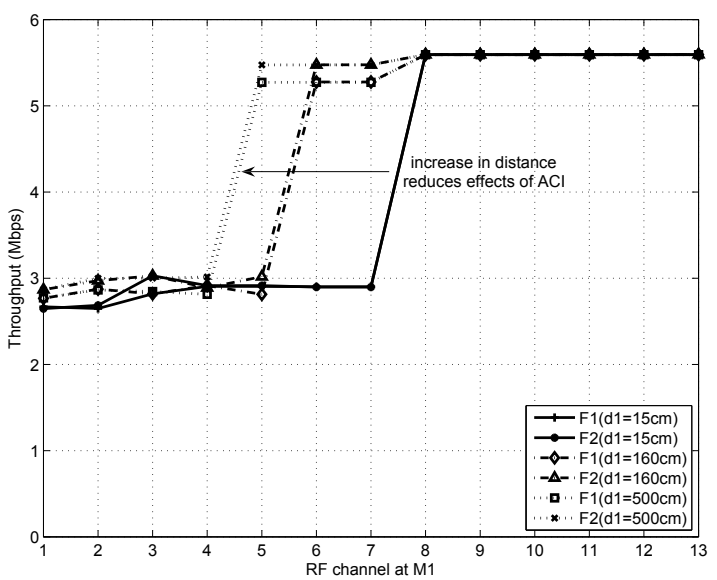

Figure 15. Simulated TX-TX scenario (single-radio, OFDM PHY, 2.4 GHz). 


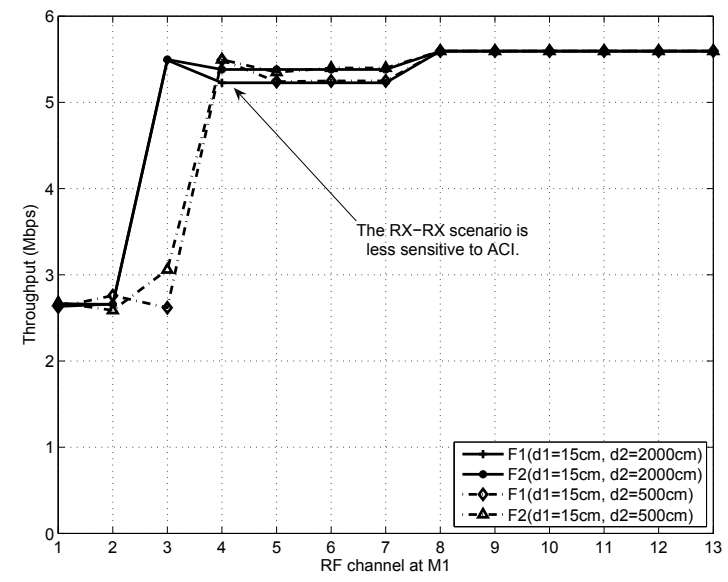

Figure 16. Simulated RX-RX scenario (single-radio, OFDM PHY, $2.4 \mathrm{GHz}$ ).

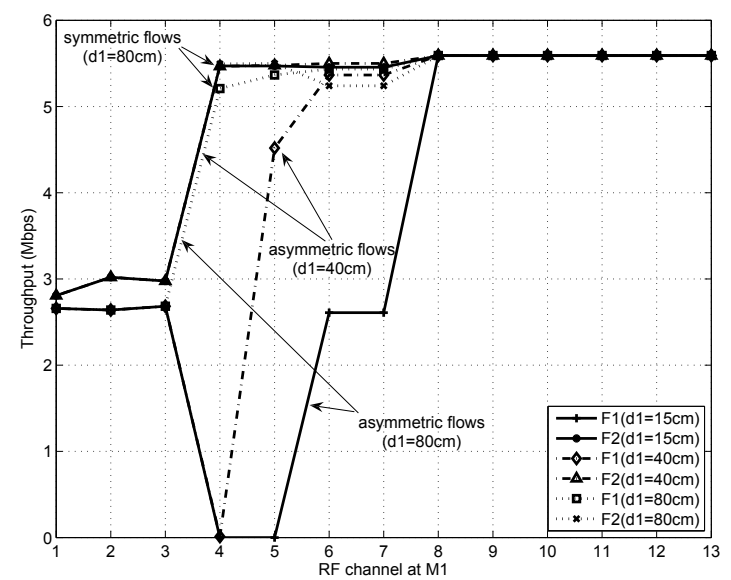

Figure 17. Simulated RX-TX scenario (single-radio, OFDM PHY, $2.4 \mathrm{GHz}$ ).

case, the throughput of the second flow is nearly zero for the channels 4 and 5. The performance improves when the distance is increased. Channels 1 and 8 are non-interfering for the distance of $15 \mathrm{~cm}$. For distances larger than $80 \mathrm{~cm}$ channel 4 is non-interfering.

\section{CONCLUSIONS}

Our measurements show that the number of available orthogonal channels in IEEE $802.11 \mathrm{~b} / \mathrm{g} / \mathrm{a}$ depends on the antenna separation, PHY modulation, RF band, traffic pattern and whether single- or multi-radios are used. If two transceivers are in close proximity to each other, as it is the default setup for multi-radio systems, the results are very dramatical. From the frequency ranges of $802.11 \mathrm{~b} / \mathrm{g}$ and $802.11 \mathrm{a}$ only one channel can be used at the same time. This results in at most two non-overlapping channels, one within the 2.4 and the other within the $5 \mathrm{GHz}$ band. Only increasing the antenna separations of the two transceivers helps. The main reason is ACI. Obviously, multi-radio systems were not the main focus during the standardization process of $802.11 \mathrm{~b} / \mathrm{g} / \mathrm{a}$. The second issue we noticed is board crosstalk and radiation leakage of the wireless cards. To overcome this problem one might use single-radio systems only. Alternatively, Ramachandran et al. [14] connected several single-radio devices via an Ultra
Wide Band backhaul or ethernet cables to form one unit, where the WiFi cards and antennas are farther separated from each other. We also showed that the OFDM PHY with 6 Mbps is more vulnerable to ACI than the DSSS PHY with $1 \mathrm{Mbps}$. This can be connected to the higher bitrate in OFDM. Furthermore, OFDM is a multi-carrier modulation whereas DSSS makes use of a single-carrier.

We hope that our detailed measurement results may help to design better multi-channel protocols which work well with contemporary 802.11 off-the-shelf hardware. Moreover, with the help of a simulator that incorporates ACI - as presented in this paper - more confident statements about the performance of various multi-channel protocols can be made.

\section{REFERENCES}

1] Adya, A., Bahl, P., Padhye, J., Wolman, A., and Zhou, L. A multi-radio unification protocol for IEEE 802.11 wireless networks. In BROADNETS'04: Proceedings of the First International Conference on Broadband Networks (Washington, DC, USA, 2004), IEEE Computer Society, pp. 344-354.

[2] BARR, R., HAAS, Z. J., AND VAn Renesse, R. JiST: an efficient approach to simulation using virtual machines. Software: Practice and Experience 35, 6 (2005), 539-576.

[3] Bicket, J., Aguayo, D., Biswas, S., And Morris, R. Architecture and evaluation of an unplanned $802.11 \mathrm{~b}$ mesh network. In MobiCom '05: Proceedings of the 11th annual international conference on Mobile computing and networking (New York, NY, USA, 2005), ACM Press, pp. $31-42$.

[4] Chebrolu, K., Raman, B., And Sen, S. Long-distance 802.11b links: performance measurements and experience. In MobiCom'06: Proceedings of the 12th annual international conference on Mobile computing and networking (New York, NY, USA, 2006), ACM Press, pp. 74-85.

[5] Cheng, C.-M., Hsiao, P.-H., Kung, H. T., and Vlah, D. Parallel use of multiple channels in multi-hop 802.11 wireless networks. In MILCOM'06: Military Communications Conference (October 2006).

[6] Cheng, C.-M., HsiaO, P.-H., Kung, H. T., And Vlah, D. Wsn07-1: Adjacent channel interference in dual-radio 802.11a nodes and its impact on multi-hop networking. In GLOBECOM'06: Global Telecommunications Conference. IEEE (San Francisco, CA, USA, 2006), pp. 1-6.

[7] Draves, R., PADHYE, J., AND ZILl, B. Routing in multi-radio, multihop wireless mesh networks. In MobiCom'04: International Conference on Mobile Computing and Networking (2004), pp. 114-128.

[8] FuXjäger, P., Valerio, D., AND Ricciato, F. The myth of nonoverlapping channels: interference measurements in IEEE 802.11. In WONS'07: Fourth Annual Conference on Wireless on Demand Network Systems and Service (2007), pp. 1-8.

[9] GASt, M. 802.11 Wireless Networks: The Definitive Guide, Second Edition. O'Reilly Media, Inc., 2005.

[10] Gupta, P., And Kumar, P. R. The capacity of wireless networks. IEEE Transactions on Information Theory 46, 2 (2000), 388-404.

[11] HAYKIn, S. Communication Systems. Wiley \& Sons, 2001.

[12] Liese, S., Wu, D., And Mohapatra, P. Experimental characterization of an $802.11 \mathrm{~b}$ wireless mesh network. In IWCMC'06: Proceedings of the 2006 International Wireless Communication and Mobile Computing Conference (New York, NY, USA, 2006), ACM Press, pp. 587-592.

[13] Mo, J., So, H.-S. W., AND Walrand, J. Comparison of multichannel MAC protocols. IEEE Transactions on Mobile Computing 7 (January 2008), 50-65.

[14] RAMACHANDRAN, K., SherifF, I., BElding, E. M., AND Almeroth, K. A multi-radio 802.11 mesh network architecture. Mobile Networks and Applications (MONET), Special Issue on Advances in Wireless Mesh Networks (2008).

[15] RAPPAPORT, T. S. Wireless Communications: Principles and Practice, 2nd ed. Prentice Hall PTR, December 2002.

[16] Robinson, J., Papagiannaki, K., Diot, C., Guo, X., And KrishNAMURTHY, L. Experimenting with a Multi-Radio Mesh Networking Testbed. In WiNMee'05: Proceedings of the First Workshop on Wireless Network Measurements (Riva del Garda, Italy, April 2005). 